\title{
Infinitely divisible probahility measures and the converse Kolmogorov inequality in Banach spaces
}

ALEJANDRO de ACOSTA* (Caracas) and JORGE D. SAMUR** (La, Plata)

Absiract. It is proved that every infinitely divisible probability measure on a separable Banach space $E$ has a representation of Khintohine's form if and only if $E$ is a space of cotype 2. This includes in particular results for the $I^{p}$ spaces, $1 \leqslant p<2$. The converse Kolmogorov inequality is generalized to the case of random vectors.

1. Introduction. Khintchine's form of the LévY-Khintchine canonical representation of the characteristic function of an infinitely divisible distribution in $R^{1}$ associates to every such distribution $\mu$ a unique triple $\left(x_{0}, a, v\right)$, where $x_{0}$ is a real number, $a$ is a non-negative real number and $v$ is a finite non-negative measure satisfying $v(\{0\})=0$ such that

(1) $\hat{\mu}(y)=\exp \left[i x_{0} y-a\left(y^{2} / 2\right)+\int K(x, y) \nu(d x)\right] \quad$ for every $y \in R^{1}$ where

$$
K(x, y)=\frac{1+x^{2}}{x^{2}}\left[\exp (i x y)-1-\frac{i x y}{1+x^{2}}\right] \quad \text { for } x \neq 0, y \in R^{1}
$$

(it is irrelevant how $K(x, y)$ is defined for $x=0$ ).

In Section 4 of this paper (Theorem 4.2) we prove that if $E$ is a separable Banach space of cotype 2 , then every infinitely divisible probability measure on $E$ has a unique representation which is the natiral generalization of (I); and, conversely, if a given separable Banach space is such that every infinitely dirisible probability measure has a representation of the form (1) (suitably generalized), then the space is of cotype 2.

* Part of this research was carried out while the first-named author was at the Departamento de Matemáticas, Facultad de Cieneias Exactas y Naturales, Universidad Nacional de Buenos Aires, Argentina.

** While carrying out this research, the second-named author was supported by a fellowship of the Consejo Nacional de Investigaciones Cientificas y Téenicas, Argentina. 
In broad outlines our proof is patterned after Khintchine's proof for the one-dimensional ease ([8], p. 76); the crucial step is the construction of the measure $\boldsymbol{\nu}$. However, the infinite-dimensional situation presents some serious difficulties, centered around proving the relative weak compactness of certain sets of measures (Theorem 4.1 ). The basic results which make it possible to handle the weak compantness problems are proved in Sections 2 and 3. Section 2 contains some general results on infinitely divisible probability measures on a separable Banach space. In Section 3 we generalize an important inequality of Kolmogorov (see e.g. [4], p. 117) to the case of Banach space-valued random vectors; this result is of independent interest.

The converse part of the representation theorem (Part (2) of Theorem 4.2) follows easily from results of Mawrey and Pisier [15].

We will now make some remarks on the connection of our work with other results in the literature. Theorem 4.2 of the present paper contains Varadhan's representation theorem in the Hilbert space case ([19]; see also [16], Chapter 6, Section 4); our techniques are different from Varadhan's in several important points. Very recently, Dettweiler ([6], Satz 2.5) has obtained a weak form of the Levy-Khintchine formula valid for infinitely divisible measures on locally convex spaces $\left.{ }^{1}\right)$; in this result the information on the "generalized Poisson" part is less specific than in our Theorem 4.2 (as should be expected, in view of part (2) of Theorem 4.2). Satz 2.5 of [6] is based on the decomposition of an infinitely divisible measure as the convolution of a Gaussian measure and a "generalized Poisson" measure (Satz 1.9 of [6]), essentially due to Tortrat ([18] and [18a]). Our work is completely independent of these results; we remark in passing that we do not even use the one-dimensional Lévy-Khintchine formula. More or less simultaneously with the final formulation of the results of the present paper, Araujo and Gine [2] have obtained a representation formula equivalent to Theorem 4.2. Their approach centers around the problem of the integrability of Lévy measures (previously studied by Araujo in [1]). The method of proof of the representation formula in [2] is based on Tortrat's decomposition and is technically different from our work, although it uses in an essential way Theorem 3.1 of the present paper.

We refer to [3] and [16] for defimitions and results on weak convergence and compactness, tightness and characteristic functionals of prob-

(1) A similar result in the case of separable Banach spaces appears in A. de Araujo's doctoral thesis, Department of Statistics, University of California at Berkeley, 1974. (See also J. Multivariate Anal. 8, 4 (1978), pp. 598-613.) ability measures on Banach spaces. All measures will be defined on the Borel $\sigma$-algebra of a separable Banach space $E$; for $x \in E, \delta_{x}$ will denote the probability measure supported by $\{x\}$. If $X$ is an $E$-valued random rector, its distribution will be denoted by $\mathscr{L}(X)$. The symbol $N$ will denote the set of natural numbers.

2. Infinitely divisible measures in Banach spaces. Let us recall that a probability measure $\mu$ on a separable Bannch space $E$ is infinitely divisible if for every $n \in N$, there exists a probability measure $\mu_{n}$ on $B$ such that $\mu_{n}^{n}=\mu\left(v^{n}\right.$ denotes the $n$th convolution power of a finite mensure $\left.v\right)$.

LEnra 2.1. Let $\mu$ be an infinitely divisible probability measure on a separable Banach space $B$. Then

(a) $\hat{\mu}(y) \neq 0$ for $a l l y \in E^{\prime}$,

(b) There exists a unique function $\Phi: E^{\prime} \rightarrow C$ such that

$\left(\mathrm{b}_{2}\right) \Phi(0)=0$,

$\left(\mathrm{b}_{2}\right) \Phi$ is sequentially $w^{*}$-continuous,

$\left(\mathrm{b}_{3}\right) \hat{\mu}=\exp \Phi$.

Proof. (a) Lret $\mu_{n}$ be sueh that $\mu_{n}^{n}=\mu$, and let $\nu_{n}=\mu_{n} * \bar{\mu}_{n}, v=\mu * \bar{\mu}$. Then $\hat{v}_{n} \geqslant 0, \hat{v} \geqslant 0$ and $\hat{\nu}_{n}=\hat{v}^{1 / n}$. Using an elementary inequality,

$$
\left[1-\hat{y}_{n}\left(y_{1}+y_{2}\right)\right] \leqslant 2\left[1-\hat{y}_{n}\left(y_{1}\right)\right]+2\left[1-\hat{\nu}_{n}\left(y_{2}\right)\right]
$$

or

$$
\left[1-\hat{\nu}^{1 / n}\left(y_{1}+y_{2}\right)\right] \leqslant 2\left[1-\hat{\nu}^{1 / n}\left(y_{1}\right)\right]+2\left[1-\hat{\nu}^{1 / n}\left(y_{2}\right)\right]
$$

for $y_{1}, y_{2} \in E^{\prime}$. Since $\hat{v}(y)>0$ implies $\lim \hat{v}^{1 / n}(y)=1$, it follows that $G=\{y: \hat{\mu}(y) \neq 0\}=\{y: \hat{\nu}(y)>0\}$ is a norm-open subgroup of $E^{\prime}$; since $0 \in G$, it follows that $G=E^{\prime}$.

(b) Fix $y \in E^{\prime}$, and define $\varrho_{y}: R^{1} \rightarrow C$ by $\varrho_{y}(t)=\hat{\mu}(t y)\left(t \in R^{1}\right)$. By [4], p. 241, there exists a unique function $\lambda_{y}: R^{1} \rightarrow C$ such that $\lambda_{y}(0)=0, \lambda_{y}$ is continuous and $\varrho_{y}=\exp \left(\lambda_{y}\right)$. Define $\Phi(y)=\lambda_{y}(1)$. Then $\Phi(0)=0$ and $\hat{\mu}(y)=\varrho_{y}(1)=\exp [\Phi(y)]\left(y \in E^{\prime}\right)$.

We show now that $\Phi$ is sequentially $w^{*}$-continuous. In fact, $y_{n} \stackrel{w^{*}}{\rightarrow} y$ implies $\mu \circ y_{n}^{-1} \stackrel{w}{\rightarrow} \mu \circ y^{-1}$, hence $\varrho_{y_{n}}(t)=\hat{\mu}\left(t y_{n}\right)=\left(\mu \circ y_{n}^{-1}\right)^{\wedge}(t)$ converges to $\left(\mu \circ y^{-1}\right)^{\wedge}(t)=\hat{\mu}(t y)=\varrho_{y}(t)$ iwiformly on compact intervals of $R^{1}$. By [4], p. 242, it follows that $\Phi\left(y_{n}\right)=\lambda_{y_{n}}(1)$ converges to $\lambda_{y}(1)=\Phi(y)$.

To prove uniqueness, let $\Phi_{1}, \Phi_{2}$ be two functions satisfying $\left(\mathrm{b}_{1}\right)$, $\left(\mathrm{b}_{2}\right),\left(\mathrm{b}_{3}\right)$. Then for all $y \in E^{\prime}, \exp \left[\Phi_{1}(y)\right]=\exp \left[\Phi_{2}(y)\right]$, which implies $\Phi_{1}(y)-\Phi_{2}(y)=(2 \pi i) k(y)$, with $k(y)$ an integer. The function $k$ is normcontinuous and $k(0)=0$; hence $k(y)=0$ for all $y \in E^{\prime}$. 
Let us remark that in part (b) of Lemma 2.1 we have only used the fact that $\hat{\mu}$ does not vanish (the infinite divisibility of $\mu$ is not otherwise relevant).

COROLLART 2.1. Let $\mu$ be an infinitely divisible probability measure on a separable Banach space. Then for each $n \in N, \mu$ has a unique n-th convolution root.

Proof. Assume $\mu_{n}^{n}=\mu$; then $\hat{\mu}_{n}^{n}=\hat{\mu}$. Since neither $\hat{\mu}$ nor $\hat{\mu}_{n}$ vanish, there exist unique functions $\Phi, \Phi_{n}$ satisfying $\left(b_{1}\right)$ and $\left(b_{2}\right)$ of Lemma 2.1, and such that $\hat{\mu}=\exp \Phi, \hat{\mu}_{n}=\exp \Phi_{n}$. It follows that $n \Phi_{n}=\Phi$; hence $\hat{\mu}_{n}=\exp [\Phi / n]$. This proves the uniqueness of $\mu_{n}$.

We recall the following result, to be used in Lemma 2.2 : if $\left\{\mu_{n}: n \in N\right\}$ is a sequence of probability measures on a separable Banach space $E$ such that (1) $\left\{\mu_{n}: n \in N\right\}$ is relatively shift-compact and (2) $\hat{\mu}_{n 2}$ converges uniformly on the balls of $E^{\prime}$ to a function $g$, then there exists a probability measure $\mu$ on $E$ such that $\mu_{n} \stackrel{w}{\rightarrow} \mu$ and $\hat{\mu}=g$. (This is proved as Theorem 4.5, Chapter 6 of [16].)

LEMMA 2.2. Let $\mu$ be an infinitely divisible probability measure on a separable Banach space, and let $\mu_{n \text { be }}$ its $n$-th convolution root $(n \in N)$. If $p$ is a positive integer, then $\left\{\mu_{n}^{k}: n \in N, k \in N, k \leqslant p n\right\}$ is relatively compaot for the weak topology.

Proof. Let $\mathscr{K}=\left\{\mu_{n}^{k}: n \in N, k \in N, k \leqslant p n\right\}$. Since $\mu_{n}^{k} * \mu_{n}^{(p n-k)}=\mu_{n}^{p n}$ $=\left(\mu_{n}^{n}\right)^{p}=\mu^{p}$, it follows from [16] (Theorem 2.2, Chapter 3) that $\mathscr{K}$ is relatively shift-compact. Let $\left\{\mu_{n_{j}}^{k_{j}}: j \in N\right\}$ be a sequence in $\mathscr{K}$. Since $\left(k_{j} / n_{j}\right) \leqslant p$, there exists a subsequence $\left(k_{j^{\prime}}, n_{j^{\prime}}\right)$ which conterges to a real number $t \in[0, p]$. Since $\left(\mu_{n}^{k}\right)^{\wedge}=\exp [(k / n) \Phi]$ (where $\Phi$ is defined as in Lemma 2.1) and $\Phi$ is bounded over the balls of $Z^{\prime}$, it easily follows that $\left(\mu_{n_{j^{\prime}}}^{K_{j^{\prime}}}\right)^{\wedge}$ converges to $\exp [t \Phi]$ uniformly on the balls of $E^{\prime}$. By the result quoted above, there exists a probability measure $y$ such that $\hat{\nu}=\exp [t \Phi]$

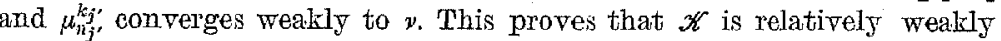
compact.

Let us recall that if $\boldsymbol{y}$ is a finite sigmed measure on a separable Banach space, then the exponential of $y$ is defined by $\exp v=\sum_{n=0}^{\infty} \frac{y^{n}}{n !}$, where $v^{0}=\delta_{0}$; the series converges in the total variation norm.

THEOREM 2.1. Let $\mu$ be an infinitely divisible probability measure on a separable Banach space, and let $\mu_{n}$ be its $n$-th convolution root $(n \in N)$. Then

(a) $\mu_{n} \stackrel{n}{\rightarrow} \delta_{0}(n \rightarrow \infty)$

(b) $\exp \left[n\left(\mu_{n}-\delta_{0}\right)\right] \stackrel{v s}{\rightarrow} \mu(n \rightarrow \infty)$.

Proof. (a) Using the expression $\hat{\mu}_{n}=\exp [\Phi / n]$ (where $\Phi$ is as in Lemma 2.1), it is readily proved that $\hat{\mu}_{n} \rightarrow 1(n \rightarrow \infty)$ uniformly over the balls of $E^{\prime}$. Since $\mu_{n}^{n}=\mu$, it follows from [16] (Theorem 2.2, Chapter 3) that $\left\{\mu_{n}: n \in N\right\}$ is relatively shift-compact. By the result quoted before Lemma 2.2, we conclude that $\mu_{n} \stackrel{w}{\rightarrow} \delta_{0}$.

(b) Let $\lambda_{n}=\exp \left[n\left(\mu_{n 2}-\delta_{0}\right)\right]$. We will prove

(I) $\hat{\lambda}_{n}(y) \rightarrow \hat{\mu}(y)(n \rightarrow \infty)$ for each $y \in E^{\prime}$,

(II) $\left\{\lambda_{n}: n \in N\right\}$ is tight.

It follows that $\lambda_{n} \stackrel{w}{\rightarrow} \mu(n \rightarrow \infty)$.

To prove (I), observe first that $\hat{\lambda}_{n}=\exp \left[n\left(\hat{\mu}_{n}-1\right)\right]=\exp [n \times$ $\times(\operatorname{crp}[\Phi / n]-1)]$

We hare, for $y \in E^{\prime}$

$$
\begin{aligned}
|n(\exp [\Phi(y) / n]-1)-\Phi(y)| & =\left|n \sum_{k=2}^{\infty} \frac{[\Phi(y) / n]^{k}}{k !}\right| \\
& \leqslant n \cdot n^{-2} \sum_{k=2}^{\infty} \frac{|\Phi(y)|^{k}}{k !} \leqslant n^{-1} \exp (|\Phi(y)|) .
\end{aligned}
$$

Therefore, $\hat{\lambda}_{n}(y) \rightarrow \exp [\Phi(y)]=\hat{\mu}(y)$ for each $y \in E^{\prime}$ (in fact, the conrergence is uniform on the balls of $E^{\prime}$ ).

For the proof of (II) we shall need the following elementary fact: $\lim _{n \rightarrow \infty} \exp (-n) \sum_{k=0}^{m_{n}} \frac{n^{k}}{k !}=1$. To prore it, let $\left\{\xi_{j}: j \in N\right\}$ be independent random rariables with $\mathscr{L}\left(\xi_{j}\right)=$ Poisson with parameter $1(j \in N), S_{n}=$ $\sum_{j=1}^{n} \xi_{j}$. It is a consequence of the weak law of large numbers that for any $x>1, P\left[S_{n} / n \leqslant x\right] \rightarrow 1(n \rightarrow \infty)$. But $\mathscr{L}\left(S_{n}\right)=$ Poisson with parameter $n$. Therefore, as $n \rightarrow \infty$

$$
\exp (-n) \sum_{k=0}^{2 n} \frac{n^{k}}{k !}=P\left[S_{n} \leqslant 2 n\right] \rightarrow 1
$$

By Lemma 2.2, for ant $\eta<1$ there exists a compact set $K \subset D$ such that $\mu_{n}^{k}(K)>\eta$ for anr pair $(n, k)$ of positive integers such that $k \leqslant 2 n$. Choose $n_{0}$ so that $n \geqslant n_{0}$ implies $\exp (-n) \sum_{k=0}^{2 n} \frac{n^{k}}{k !}>\eta$. Then for $n \geqslant n_{0}$

$$
\begin{aligned}
\lambda_{n}(K) & =\exp (-n) \sum_{k=0}^{\infty} \frac{n^{k}}{k !} \mu_{n}^{k}(K) \\
& \geqslant \exp (-n) \sum_{k=0}^{2 n} \frac{n^{k}}{k !} \mu_{n}^{k}(K)>\eta^{2} .
\end{aligned}
$$

The proof that $\left\{\hat{\lambda}_{n}: n \in N\right\}$ is tight is completed by a standard argument. 
Remark 2.1. We shall not use in the sequel the full strength of Theorem 2.1 (b). We include this result becanse of its obvious interest; it provides a canonical way of expressing an arbitrary infinitely divisible probability measure as the weak limit of a sequence of measures of exponential type.

The following result $\left({ }^{2}\right)$ (which will not be used in this paper) may be proved in a similar way.

THEOREM 2.2. Let $\left\{\mu_{n}: n \in N\right\}$ be a sequence of probability measures on a separable Banach space. Let $\left\{k_{n}: n \in N\right\}$ be a sequence of positive integers such that $k_{n} \rightarrow \infty$. Suppose $\left\{\mu_{n}^{k_{n}}: n \in N\right\}$ is relatively compact. Then

(a) $\mu_{n 2} \stackrel{w}{\rightarrow} \delta_{0}(n \rightarrow \rightarrow \infty)$,

(b) If $\mu_{n}$ is symmetric $(n \in N)$, then $\left\{\exp \left[k_{n}\left(\mu_{n}-\delta_{0}\right)\right]: n \in N\right\}$ is relatively compact.

Remark 2.2. Let $\left\{\mu_{n j}: n \in N, j=1, \ldots, k_{n}\right\}$ be a triangular array of probability measures on $E$. It is well known that the relation between weak compactness and weak convergence properties of $\left\{\prod \mu_{n j}: n \in N\right\}$ and the corresponding properties of $\left\{\exp \left[\sum_{j}\left(\mu_{n j}-\delta_{0}\right)\right]: n \in N\right\}$ plays a key role in the general central limit problem for triangular arrays; this fact has been emphasized in [14]. Theorem 2.2 (which deals with the case of identically distributed rows) is of some interest in view of a counterexample of Le Cam ([14], p. 240) for general triangular arrays.

Our next proposition generalizes an inequality in Feller's book ([7], p. 149) and is essential for the proof of some weak compactness results (Theorems 2.3 and 5.1). Let us recall that a generalized seminorm $q$ on a real vector space $E$ is a function $q: E \rightarrow[0, \infty]$ such that $q(x+y)$ $\leqslant q(x)+q(y), q(\lambda x)=|\lambda| q(x) \quad\left(x \in E, y \in E, \lambda \in R^{\mathrm{I}}\right)$.

LEMLA 2.3. Let $E$ be a separable Banach space, and suppose $q$ is a neasurable generalized seminom on $E$. Let $\left\{X_{j}: j=1, \ldots, n\right\}$ be independent symmetric E-valued random vectors, $S_{n}=\sum_{j=1}^{n} X_{j}$. Then for every $t>0$ such that $P\left[q\left(S_{n}\right)>t\right]<(1 / 2)$,

$$
\sum_{j=1}^{n} P\left[q\left(X_{j}\right)>t\right] \leqslant-\log \left(1-2 P\left[q\left(S_{n}\right)>t\right]\right) .
$$

Proof. Let $A_{k}=\left[q\left(X_{j}\right) \leqslant t, j=1, \ldots, k-1 ; q\left(X_{k}\right)>t\right], \quad A=$ $\left[\sup _{1 \leqslant k \leqslant n} q\left(X_{k}\right)>t\right]$. Then $\left\{A_{k}: k=1, \ldots, n\right\}$ is disjoint and $A=\bigcup A_{k}$. Fix $k$, and let $Z=2 X_{k}-S_{n}$. Then $\left[q\left(S_{n}\right) \leqslant t\right] \cap[q(Z) \leqslant t] \subset\left[q\left(X_{k}\right) \leqslant t\right]$,

(') A. de Araujo communicated to us that he was independently aware of this and consequently

$$
A_{k}=\left(A_{k} \cap\left[q\left(S_{n}\right)>t\right]\right) \cup\left(A_{k} \cap[q(Z)>t]\right] .
$$

Let $Y_{j}=-X_{j}$ for $j \neq k,=X_{k}$ for $j=k$. The symmetry and independence of $\left\{X_{j}: j=1, \ldots, n\right\}$ implies: $\mathscr{L}\left(Y_{1}, \ldots, Y_{n}\right)=\mathscr{L}\left(X_{1}, \ldots\right.$ $\left.\ldots, X_{n}\right)$. Observe that $A_{k}=\left[q\left(Y_{j}\right) \leqslant t, j=1, \ldots, k-1 ; q\left(Y_{k}\right)>t\right]$ and $Z=\sum_{j=1}^{n} Y_{j} ;$ it follows that $P\left(A_{k} \cap\left[q\left(S_{n}\right)>t\right]\right)=P\left(A_{k} \cap[q(Z)>t]\right)$, and we obtain from (1)

$$
P\left(A_{k}\right) \leqslant 2 P\left(A_{k} \cap\left[q\left(S_{n}\right)>t\right]\right)
$$

Adding over $k$,

Now

$$
P(\mathcal{A}) \leqslant 2 P\left[q\left(S_{n}\right)>t\right]
$$

$$
\begin{aligned}
P(A) & =1-P\left[\sup _{k} q\left(X_{k}\right) \leqslant t\right] \\
& =1-\prod_{k} P\left[q\left(X_{k}\right) \leqslant t\right] \\
& \geqslant 1-\exp \left(-\sum_{k} P\left[q\left(X_{k}\right)>t\right]\right)
\end{aligned}
$$

and therefore

$$
\sum_{k=1}^{n} P\left[q\left(X_{k}\right)>t\right] \leqslant-\log (1-P(A)) .
$$

The desired inequality follows from (2) and (3).

THEOREM 2.3. Let $\mu$ be an infinitely divisible probability measure on a separable Banach space, and let $\mu_{n}$ be its $n$-th convolution root. Then there exists a compact, convex, symmetric set $K$ such that $\left\{n \mu_{n}\left(K^{c} \cap(\cdot)\right): n \in N\right\}$ is relatively compact.

Proof. Let $v=\mu * \bar{\mu}, v_{n}=\mu_{n} * \bar{\mu}_{n}(n \in N)$. For each $n \in N$, let $\left\{X_{n j}\right.$; $j=1, \ldots, n\}$ and $\left\{X_{n j}^{\prime}: j=1, \ldots, n\right\}$ be each an independent set of $E$ valued random rectors with $\mathscr{L}\left(X_{n j}\right)=\mathscr{L}\left(X_{n j}^{\prime}\right)=\mu_{n}$; assume also that the pair of sets $\left\{X_{n j}: j=1, \ldots, n\right\}$ and $\left\{X_{n j}^{\prime}: j=1, \ldots, n\right\}$ is independent Let $S_{n}=\sum X_{n j}, S_{n}^{\prime}=\sum X_{n j}^{\prime} ;$ then $\mathscr{L}\left(S_{n}\right)=\mathscr{L}\left(S_{n}^{\prime}\right)=\mu, \mathscr{L}\left(X_{n j}-X_{n j}^{\prime}\right)$ $=v_{n}(j=1, \ldots, n)$ and $\mathscr{L}\left(S_{n}-S_{n}^{\prime}\right)=v$.

Let $O$ be a compact, convex, symmetric set such that $\mu_{n}(O) \geqslant(1 / 2)$ for all $n \in N$ (recall that $\left\{\mu_{n}: n \in N\right\}$ is tight by Theorem 2.1 (a)). Let $D$ be a compact, convex, symmetric set such that $\nu\left(D^{c}\right)<1 / 4$, and define $K=$ $C+D$. Define $\lambda_{n}=n \mu_{n}\left(K^{c} \cap(\cdot)\right)(n \in N)$; we will prove that $\left\{\lambda_{n}: n \in N\right\}$ is relatively compact. 
By the independence of $X_{n j}$ and $X_{n j}^{\prime}$

$$
\begin{aligned}
(1 / 2) P\left[X_{n j} \notin K\right] & \leqslant P\left[X_{n j}^{\prime} \in O\right] P\left[X_{n j} \notin K\right] \\
& =P\left[X_{n j}^{\prime} \in O, X_{n j} \notin K\right] \\
& \leqslant P\left[X_{n j}-X_{n j}^{\prime} \notin D\right] .
\end{aligned}
$$

Let $q$ be the Minkowski functional of $D$. Applying Lemma 2,3, we have for all $n \in N$

$$
\begin{aligned}
\left\|\lambda_{n}\right\| & =n \mu_{n}\left(K^{c}\right)=\sum_{j=1}^{n} P\left[X_{n j} \notin K\right] \leqslant 2 \sum_{j=1}^{n} P\left[X_{n j}-X_{n j}^{\prime} \notin D\right] \\
& =2 \sum_{j=1}^{n} P\left[q\left(X_{n j}-X_{n j}^{\prime}\right)>1\right] \leqslant-2 \log \left(1-2 P\left[q\left(S_{n}-S_{n}^{\prime}\right)>1\right]\right) . \\
& =-2 \log \left(1-2 v\left(D^{c}\right)\right)<2 \log 2 .
\end{aligned}
$$

We have shown that $\sup _{n}\left\|\lambda_{n}\right\|<\infty$. To prove the tightness of $\left\{\lambda_{n}: n \in N\right\}$, let $\varepsilon>0$ be given, and let $Q$ be a compact convex symmetric set such that $\nu\left(Q^{c}\right)<(1 / 2)(1-\exp (-(\varepsilon / 2)))$. Proceeding as above, we obtain, for all $n \in N$

$$
\begin{aligned}
\lambda_{n}\left((Q+O)^{c}\right) & =n \mu_{n}\left(K^{c} \cap(Q+O)^{c}\right) \\
& \leqslant n \mu_{n}\left((Q+O)^{c}\right)<\varepsilon
\end{aligned}
$$

Remark 2.3. There are other methods of proving Theorem 2.3 . One way is to apply results of Le Cam [14], besed on a concentration inequality. A modification of the proof of Theorem 4.3, Chapter 4 of [16], combined with Theorem 2.1 (b) of the present work, also yields an alternative proof. Our approach is a generalization of a method of Feller $([7]$, p. 309); for the present purposes, it seems to be simpler and more direct than either alternative we have mentioned.

3. The converse Kolmogorov inequality for Banach space valued random vectors. Let $E$ be a separable Banach space, $I \leqslant p<\infty$. Let $L^{p}(E)$ be the vector space of (equivalence classes of) $E$-valued random vectors $X$ such that $E\|X\|^{p}<\infty$, endowed with the norm $\|X\|_{p}=\left(E\|X\|^{p}\right)^{1 / p}$.

The following inequality (Theorem 2.6 in [9]) is easily proved. It plays a crucial role in Theorem 3.1.

Proposttion 3.1. Let $E$ be a separable Banach space, $1 \leqslant p<\infty$. Let $X, Y$ be independent tE-valued random vectors, $X \in L^{\nu}(E), Y \in L^{p}(E)$, $E(X)=0$. Then

$$
E\|Y\|^{p} \leqslant E\|X+Y\|^{p} .
$$

THEOREXr 3.1: Let be a separable Banach space. Let $\left\{X_{j}: j=1, \ldots, n\right\}$ be independent $\mathbb{E}$-valued randon vectors such that, for some $c \in[0, \infty),\left\|X_{j}\right\| \leqslant 0$ a.s. and $E\left(X_{j}\right)=0(j=1, \ldots, n)$. Let $S_{k}=\sum_{j=1}^{k} X_{j}(k=1, \ldots, n), p \geqslant 1$. Then for every $t>0$

$$
P\left[\sup _{1 \leqslant k \leqslant n}\left\|S_{k}\right\|>t\right] \geqslant 2^{1-p}\left[1-\frac{(t+c)^{p}+t^{p}\left(1-2^{1-p}\right)}{E\left\|S_{n}\right\|^{p}}\right] .
$$

Proof. Let $A=\left[\sup _{1 \leqslant k \leqslant n}\left\|S_{k}\right\|>t\right], A_{k}=\left[\left\|S_{j}\right\| \leqslant t\right.$ for $j=1, \ldots$ $\left.\ldots, k-1 ;\left\|S_{k}\right\|>t\right]$. Then

$$
\begin{aligned}
E\left(\left\|S_{n}\right\| \|^{p} I_{A}\right) & =\sum_{k=1}^{n} E\left(\left\|S_{n}\right\| \|^{p} I_{A_{k}}\right)=\sum_{k=1}^{n} E\left(\left\|S_{k}+\left(S_{n}-S_{k}\right)\right\|^{p} I_{A_{k}}\right) \\
& \leqslant \sum_{k=1}^{n} E\left(\left(\left\|S_{k}\right\|+\left\|S_{n}-S_{k}\right\|\right)^{p} I_{A_{k}}\right) \\
& \leqslant 2^{p-1} \sum_{k=1}^{n} E\left(\left\|S_{k}\right\|^{p} I_{A_{k}}\right)+2^{p-1} \sum_{k=1}^{n} E\left(\left\|S_{n}-S_{k}\right\|^{p} I_{A_{k}}\right)
\end{aligned}
$$

Since $\left[\left\|S_{t^{-1}-1}\right\| \leqslant t,\left\|X_{k_{i}}\right\| \leqslant c\right] \subset\left[\left\|S_{t_{t}}\right\| \leqslant t+o\right]$, it follows that

$$
\sum_{k=1}^{n} E\left(\left\|S_{k}\right\|^{p} I_{A_{k}}\right) \leqslant(t+c)^{p} \sum_{k=1}^{n} P\left(A_{k}\right)=(t+c)^{p} P(A) .
$$

On the other hand, by the independence of $I_{\mathcal{A}_{k}}$ and $\left(S_{n}-S_{k}\right)$ and Proposition 3.1, we have

and therefore

$$
\begin{aligned}
E\left(\left\|S_{n}-S_{k}\right\|^{p} I_{A_{k}}\right) & =\left(E\left\|S_{n}-S_{k}\right\|^{p}\right)\left(E\left(I_{d_{k}}\right)\right) \\
& \leqslant E\left\|S_{n}\right\|^{p} \cdot P\left(A_{k}\right)
\end{aligned}
$$

$$
\sum_{k=1}^{n} E\left(\left\|\mathcal{S}_{n}-\mathcal{S}_{k}\right\|^{p} I_{\boldsymbol{A}_{k}}\right) \leqslant E\left\|\boldsymbol{S}_{n}\right\|^{p} P(\boldsymbol{A})
$$

Thus

$$
E\left(\left\|S_{\jmath \imath}\right\|^{p} I_{A}\right) \leqslant 2^{p-1}\left[(t+c)^{p}+E\left\|S_{n}\right\|^{p}\right] P(A) .
$$

Since obvionsly $E\left(\left\|S_{n}\right\|^{p} I_{\mathcal{A}^{c}}\right) \leqslant t^{p}(1-P(A))$, we have

$$
\mathbb{E}\left\|S_{n}\right\|^{p} \leqslant t^{p}+\left[2^{p-1}(t+e)^{p}+2^{p-1} E\left\|S_{n}\right\|^{p}-t^{p}\right] P(A) .
$$

Inequality $(*)$ follows now by elementary manipulations.

COROLLART 3.1. Let $t$ be a separable Banach space. Let $\left\{X_{j}: j=1, \ldots\right.$ $\ldots, n\}$ be independent $E$-valued symmetric random vectors such that for some $c \in[0, \infty),\left\|X_{j}\right\| \leqslant 0$ a.s. $(j=1, \ldots, n)$. Let $s_{n}=\sum_{j=1}^{n} X_{j}, p \geqslant 1$. Then for 
every $t>0$

$$
\text { (**) } \quad P\left[\left\|S_{n}\right\|>t\right] \geqslant 2^{-p}\left[1-\frac{(t+c)^{p}+t^{p}\left(1-2^{1-p}\right)}{E\left\|S_{n}\right\|^{p}}\right] \text {. }
$$

Proof. According to P. Léry's inequality for Banach space valued symmetric random vectors $([13], p .12), P\left[\sup _{1}\left\|S_{k}\right\|>t\right] \leqslant 2 P\left[\left\|S_{n}\right\|>t\right]$. Inequality $(* *)$ follows at once from this fact and Theorem 3.1.

Remark. Let $q$ be a continuous seminorm on a separable Banach space $E$. Then (*) and (**) hold for $q$ instead of $\|\cdot\|$, with the same proof (using the appropriate versions of Proposition 3.1 and P. Lévy's inequality).

4. Infinitely divisible measures in spaces of cotype 2. Let $\left\{\varepsilon_{j}: j \in N\right\}$ be a Bernoulli sequence; that is, $\left\{\varepsilon_{j}\right\}$ is a sequence of independent random variables with $P\left[\varepsilon_{j}=1\right]=P\left[\varepsilon_{j}=-1\right]=1 / 2$. We recall that a Banach space $E$ is of cotype 2 if there exists a constant $C>0$ such that for every finite set $\left\{x_{1}, \ldots, x_{n}\right\} \subset E$,

$$
\sum_{j=1}^{n}\left\|x_{j}\right\|^{2} \leqslant \dot{C D}\left\|\sum_{j=1}^{n} \varepsilon_{j} x_{j}\right\|^{2}
$$

(See [15].)

If $Z$ is a separable Banach space of cotype 2 , there exists a constant $M>0$ such that if $X_{j} \in L^{2}(E), E\left(X_{j}\right)=0(j=1, \ldots, n)$ and $\left\{X_{j}\right.$ : $j=1, \ldots, n\}$ are independent, then

$$
\sum_{j=1}^{n} E\left\|X_{j}\right\|^{2} \leqslant M E\left\|\sum_{j=1}^{n} X_{j}\right\|^{2} .
$$

(This is proved as Theorem 2.1 of [10].) It is known that the $L^{p}$ spaces, $1 \leqslant p \leqslant 2$ are of cotype 2 .

Let $E$ be a separable Banach space of cotype 2. If the hypotheses of Theorem 3.1 are fulfilled, then inequality $(*)$ implies (for appropriate $t$ )

$$
\sum_{j=1}^{n} E\left\|X_{j}\right\|^{2} \leqslant M\left[(t+c)^{2}+\left(t^{2} / 2\right)\right]\left(1-2 P\left[\sup _{1 \leqslant k \leqslant n}\left\|S_{k}\right\|>t\right]\right)^{-1}
$$

analogously, if the assumptions of Corollary 3.1 are fulfilled, then inequality (**) implies (for appropriate $t$ )

$$
\sum_{j=1}^{n} E\left\|X_{j}\right\|^{2} \leqslant M\left[(t+c)^{2}+\left(t^{2} / 2\right)\right]\left(1-4 P\left[\left\|S_{n}\right\|>t\right]\right)^{-1} .
$$

THEOREM 4.1. Let $\mu$ be an infinitely divisible probability measure on a separable Banach space of cotype 2 , and let $\mu_{n}$ be its $n$-th convolution root.
Define the measure $\nu_{n}$ by

$$
\nu_{n}(d x)=\frac{\|x\|^{2}}{1+\|x\|^{2}} n \mu_{n}(d x)
$$

Then $\left\{v_{n}: n \in N\right\}$ is relatively compact.

Proof. We will show that sup $\left\|p_{n}\right\|<\infty$ and $\left\{y_{n}: n \in N\right\}$ is tight. First we prove that for any $r \in[0, \infty)$, if $B_{r}=\{x \in E:\|x\| \leqslant r\}$,

$$
\sup _{n} n \int_{B_{r}}\|x\|^{2} \mu_{n}(d x)<\infty \text {. }
$$

The meastures $\sigma_{n}=(1 / 2)\left(\mu_{n}+\bar{\mu}_{n}\right)$ are symmotric and the sequence $\left\{\sigma_{n}^{n}: n \in N\right\}$ is relatively compact. In fact, $\left\{\mu_{n}^{k}: k \leqslant n, k \in N, n \in N\right\}$ and $\left\{\bar{\mu}_{n}^{k}: k \leqslant n, k \in N, n \in N\right\}$ are relatively compact by Lemma 2.2 . If $K$ is a compact set such that $\left(\mu_{n}^{k} * \bar{\mu}_{n}^{h}\right)\left(K^{c}\right)<\varepsilon$ for all $n \in N, k \leqslant n, h \leqslant n$, then

$$
\sigma_{n}^{n}\left(K^{c}\right)=2^{-n} \sum_{k=0}^{n}\left(\begin{array}{l}
n \\
k
\end{array}\right)\left(\mu_{n}^{k} * \bar{\mu}_{n}^{n-k}\right)\left(K^{c}\right)<\varepsilon 2^{-n} \sum_{k=0}^{n}\left(\begin{array}{l}
n \\
k
\end{array}\right)=\varepsilon .
$$

For each $n \in N$, let $Y_{n j}(j=1, \ldots, n)$ be independent $E$-valued random vectors with $\mathscr{L}\left(Y_{n j}\right)=\sigma_{n}, T_{n}=\sum_{j=1}^{n} Y_{n j}$. For $r>0$, define $X_{n j}^{(r)}=Y_{n j} T_{B_{r}}\left(Y_{n j}\right)$, $S_{n}^{(r)}=\sum_{j=1}^{n} X_{n j}^{(r)}$. Since $\left\{X_{n j}^{(r)}: j=1, \ldots, n\right\}$ are independent and symmetric and $\left\|X_{n j}^{(r)}\right\| \leqslant r$ a.s., we obtain from inequality (II)

(2) $\quad n \int_{B_{r}}\|x\|^{2} \mu_{n}(d x)=n \int_{B_{r}}\|x\|^{2} \sigma_{n}(d x)^{\circ}=n E\left\|X_{n}^{(r)}\right\|^{2}$

$$
=\sum_{j=1}^{n} E\left\|X_{n j}^{(r)}\right\|^{2} \leqslant M\left[(t+r)^{2}+\left(t^{2} / 2\right)\right]\left(1-4 P\left[\left\|S_{n}^{(r)}\right\|>t\right]\right)^{-1}
$$

whenever $t>0$ satisfies $P\left[\left\|S_{n}^{(r)}\right\|>t\right]<1 / 4$.

Since $\mathscr{L}\left(T_{n}\right)=\sigma_{n}^{n}$, we may choose $r$ arbitrarily large and such that for all $n \in N, P\left[\left\|T_{n}\right\|>r\right]<1 / 24$. Then

$$
\begin{aligned}
P\left[\left\|S_{n}^{(r)}\right\|>r\right] \leqslant P\left[\left\|S_{n}^{(r)}\right\|\right. & \left.>r \text { and }\left\|Y_{n j}\right\| \leqslant r, j=1, \ldots, n\right]+ \\
& +P\left[\sup _{1 \leqslant j \leqslant n}\left\|Y_{n j}\right\|>r\right] \leqslant 3 P\left[\left\|T_{n}\right\|>r\right]<1 / 8
\end{aligned}
$$

by inequality (2) in the proof of Lemma 2.3. By puting $t=r$, we obtain from (2):

$$
\sup _{n} n \int_{B_{r}}\|x\|^{2} \mu_{n}(d x) \leqslant M\left[(2 r)^{2}+\left(r^{2} / 2\right)\right] \cdot 2=M\left(9 r^{2}\right)
$$

This proves claim (1). 
By Theorem 2.3, there exists a compact (convex, symmetric) set such that $\left\{n \mu_{n}\left(K^{c} \cap(\cdot)\right): n \in N\right\}$ is relatively compact. Ohoose $r$ so that $K \subset B_{r}$. Then

$$
\left\|v_{n}\right\|=\int \frac{\|x\|^{2}}{1+\|x\|_{1}^{2}} n \mu_{n}(d x) \leqslant n \int_{B_{r}}\|x\|^{2} \mu_{n}(d x)+n \mu_{n}\left(B_{r}^{c}\right)
$$

and consequently $\sup _{n}\left\|p_{n}\right\|<\infty$.

To prove the tightness of $\left\{\nu_{n}: n \in N\right\}$, let $D$ be a compact set such that $n \mu_{n}\left(K^{c} \cap D^{c}\right)<\varepsilon$ for all $n \in N$. If $Q=K \cup D$, then for all $n \in N$

$$
v_{n}\left(Q^{c}\right)=\int_{Q^{c}} \frac{\|x\|^{2}}{1+\|x\|^{2}} n \mu_{n}(d x) \leqslant n \mu_{n}\left(Q^{c}\right)<\varepsilon .
$$

Remark. An alternative proof of claim (1) in the proof of Theorem 4.1 may be constructed by using Theorem 2.1 (b) and following the line of proof of Theorem 4.6, Chapter 6 of [16]. The crucial inequality (II) above must be nsed at the appropriate point.

The following lemma is well-known; it follows from Prohorov's extension theorem for eylinder measures ([3], Exposé No. 7) and the fact that on a separable Banach space every probability measure is a Radon mear sure.

LEMMA 4.1. Let $\mu, \nu, \lambda$ be oylinder measures on a separable Banach space and assume $\mu=\nu * \lambda$.

(1) If $\nu$ and $\lambda$ are symmetric and $\mu$ is a measure, then $\nu$ and $\lambda$ are measures.

(2) If $\mu$ and $y$ are measures, then $\lambda$ is a measure.

Let $E$ be a separable Banach space. Define

$$
K(x, y)= \begin{cases}\frac{1+\|x\|^{2}}{\|x\|^{2}}\left[\exp (i\langle x, y\rangle)-1-\frac{i\langle x, y\rangle}{1+\|x\|^{2}}\right], & x \neq 0, y \in E^{\prime} \\ 0, & x=0, y \in E^{\prime}\end{cases}
$$

For fixed $y \in E^{\prime}, \boldsymbol{K}(\cdot, y)$ is bounded; it is continuous on $E-\{0\}$. If $\operatorname{dim} E=1$ (to simplify, assume $E=R^{1}$ ), then for any $y \in E^{\prime} \quad \lim K(x, y)$ $\underset{\substack{x \rightarrow 0 \\ x \rightarrow 0}}{\substack{x \rightarrow 0 \\ \hdashline}}$

exists (and is equal to $\left(-y^{2} / 2\right)$, which is really the natural definition of $K(0, y)$ in the one-dimensional case); however, the limit does not exist for $y \neq 0$ if $\operatorname{dim} E>1$. This explains a slight technical complication in the proof of Theorem 4.2, as compared to Khintchine's proof in the onedimensional case (see [8], p. 76 ).

LEMLA 4.2. Let $v$ be a finite non-negative measure on a separable Banaoh space $E$, such that $v(\{0\})=0$. Let $\Phi(y)=\exp \left[\int K(x, y) \nu(d x)\right], y \in \mathbb{E}^{\prime}$. Then $\Phi(0)=1$ and $\Phi$ is positive definite and sequentially $w^{*}$-continuous.
Proof. It is obvious that $\Phi(0)=1$; the fact that $\Phi$ is sequentially

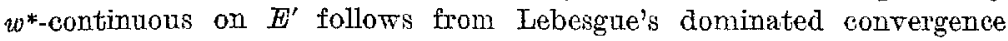
theorem.

To prove that $\Phi$ is positive definite, let $\left\{\lambda_{n}: n \in N\right\}$ be a sequence of non-negative measures of finite support disjoint from $\{0\}$ such that $\lambda_{n} \stackrel{w}{\rightarrow} y$. Each $\lambda_{n}$ is of the form $\lambda_{n}=\sum_{j} \alpha_{n j} \delta_{x_{n j}}$ for certain $\alpha_{n j} \in R^{+}, x_{n j} \in E-\{0\}$.

$$
z_{n}=-\sum_{j}\left\|x_{n j}\right\|^{-2} x_{n j}, \quad \beta_{n j}=a_{n j}\left(\frac{1+\left\|x_{n j}\right\|^{2}}{\left\|x_{n j}\right\|^{2}}\right) .
$$

Then

$$
\begin{gathered}
\left(\exp \left[\sum_{j} \beta_{n j}\left(\delta_{x_{n j}}-\delta_{0}\right)\right] * \delta_{z_{n}}\right)^{\wedge}(y) \\
\quad=\exp \left[\sum_{j} \alpha_{n j}\left(\exp \left(i\left\langle x_{n j}, y\right\rangle\right)-1-\frac{i\left\langle x_{n j}, y\right\rangle}{1+\left\|x_{n j}\right\|^{2}}\right) \frac{1+\left\|x_{n j}\right\|^{2}}{\left\|x_{n j}\right\|^{2}}\right] \\
=\exp \left[\int K(x, y\rangle \lambda_{n 2}(d x)\right] \rightarrow \exp \left[\int K(x, y) v(d x)\right]
\end{gathered}
$$

for each $y \in \mathcal{B}^{\prime}$, since $v(\{x: K(\cdot, y)$ is discontinuous at $x\})=0$. Since positive definiteness is preserved by passage to the limit, the result follows.

THEOREM 4.2. Let $E$ be a separable Banach space.

(1) If $E$ is of cotype 2, then for every infinitely divisible probability measure $\mu$ on $E$ there exist $x_{0} \in E$, a centered Gaussian measure $\gamma$ on $E$ and a finite non-negative measure $y$ on $\mathbb{Z}$ satisfying $v(\{0\})=0$, such that

(a) There exists a probability measure $\varrho$ on $\mathbb{E}$ with $\hat{\varrho}(y)=\exp \left[\int K(x, y) \times\right.$ $\times v(d x) \mid, y \in E^{\prime}$.

\section{(b) $\mu=\delta_{x_{0}} * \gamma * \varrho$.}

The triple $\left(x_{0}, \gamma, \nu\right)$ (subject to the stated conditions) is unique.

(2) Conversely, suppose that for every infinitely divisible probability measure $\mu$ on $D$ there exists a triple $\left(x_{0}, \gamma, v\right)$ with the stated properties such that (a) and (b) hold. Then $E$ is of cotype 2 . by

Proof. (1) We will use an auxiliary function $B: E \times D^{\prime} \rightarrow C$, definer $B(x, y)=$

$$
\begin{cases}\frac{1+\|x\|^{2}}{\|x\|^{2}}\left[\exp (i\langle x, y\rangle)-1-\frac{i\langle x, y\rangle}{1+\|x\|^{2}}+(1 / 2) \frac{|\langle x, y\rangle|^{2}}{\left(1+\|x\|^{2}\right)^{2}}\right], & x \neq 0, y \in E^{\prime} \\ 0, & x=0, y \in \mathbb{E}^{\prime}\end{cases}
$$


$B$ has the following properties: for $y \in E^{\prime}, B(\cdot, y)$ is a bounded continuous function on $E, B(x,-y)=B(-x, y)\left(x \in E, y \in E^{\prime}\right)$ and

$$
B(x, y)=K(x, y)+(1 / 2) \frac{|\langle x, y\rangle|^{2}}{\|x\|^{2}\left(1+\|x\|^{2}\right)} .
$$

Let $\mu_{n}$ be the $n$th convolution root of $\mu$, and let

$$
\begin{gathered}
f_{n}(y)=\int \frac{\langle x, y\rangle}{1+\|x\|^{2}} n \mu_{n}(d x), \quad g_{n}(y)=\int \frac{|\langle x, y\rangle|^{2}}{\left(1+\|x\|^{2}\right)^{2}} n \mu_{n}(d x) \quad\left(y \in E^{\prime}\right) \\
y_{n}(d x)=\frac{\|x\|^{2}}{1+\|x\|^{2}} n \mu_{n}(d x) .
\end{gathered}
$$

Then one can write, for all $y \in E^{\prime}$

$$
\begin{aligned}
n\left[\hat{\mu}_{n}(y)-1\right] & =\int[\exp (i\langle x, y\rangle)-1] n \mu_{n}(d x) \\
& =i f_{n}(y)-(1 / 2) g_{n}(y)+\int B(x, y) v_{n}(d x) .
\end{aligned}
$$

Therefore

and

$$
\begin{aligned}
n\left[\hat{\mu}_{n}(y)-1\right] & =n\left[\hat{\mu}_{n}(-y)-1\right] \\
& =-i f_{n}(y)-(1 / 2) g_{n}(y)+\int B(x, y) \vec{v}_{n}(d x)
\end{aligned}
$$

$$
n\left[\left(\mu_{n}+\bar{\mu}_{n}\right)^{\wedge}(y)-2\right]=-g_{n}(y)+\int B(x, y)\left(v_{n}+\bar{\nu}_{n}\right)(d x) .
$$

Theorem 2.1 implies that $\exp \left[n\left(\hat{\mu}_{n}(y)-1\right)\right] \rightarrow \hat{\mu}(y) \quad\left(y \in E^{\prime}\right)$, hence $\exp \left[n\left(\left(\mu_{n}+\bar{\mu}_{n}\right)^{\wedge}(y)-2\right)\right] \rightarrow(\mu * \bar{\mu})^{\wedge}(y)$. By Theorem 4.1, there exist a subsequence $\left\{v_{n_{k}}\right\}$ of $\left\{v_{n}\right\}$ and a finite non-negative measure $y^{\prime}$ on $E$ such that $\nu_{n_{k}} \stackrel{w}{\rightarrow} v^{\prime}$. Consequently $\nu_{n_{k}}+\bar{\nu}_{n_{k}} \stackrel{w}{\rightarrow} \nu^{\prime}+\bar{v}^{\prime}$ and from (3) we obtain, letting $k \rightarrow \infty$ :

and

$$
g(y)=\lim _{k} g_{n_{k}}(y) \quad \text { exists }
$$

$$
(\mu * \bar{\mu})^{\wedge}(y)=\exp \left[-g(y)+\int B(x, y)\left(\nu^{\prime}+\bar{\gamma}^{\prime}\right)(d x)\right] .
$$

Let $y=\nu^{\prime}-v^{\prime}(\{0\}) \delta_{0}$. One can write

(6)

$$
\int B(x, y) \nu^{\prime}(d x)=\int K(x, y) v(d x)+(1 / 2) h(y)
$$

where

$$
h(y)=\int \frac{|\langle x, y\rangle|^{2}}{\|x\|^{2}\left(1+\|x\|^{2}\right)} v(d x)
$$

Let $\psi=g-h$. We will prove now that $\psi(y) \geqslant 0$ for all $y \in \mathbb{F}^{r}$. Fix $y \in E^{\prime}$ and let $u: E \rightarrow R^{1}$ be the function

$$
u(x)= \begin{cases}\frac{|\langle x, y\rangle|^{2}}{\|x\|^{2}\left(1+\|x\|^{2}\right)}, & x \neq 0, \\ 0, & x=0 .\end{cases}
$$

Then $u$ is lower semicontinuous, bounded and

Fence

$$
g_{n}(y)=\int u d v_{n}, \quad h(y)=\int u d \nu^{\prime} .
$$

$$
h(y)=\int u d \nu^{\prime} \leqslant \frac{\lim }{h_{k}} \int u d v_{n_{k t}}=\lim _{k} g_{n_{k}}(y)=g(y)
$$

since $\nu_{u_{k}} \stackrel{w}{\rightarrow} v^{\prime}$.

We obtain now from (5) and (6)

$$
(\mu * \bar{\mu})^{\wedge}(y)=\exp \left[-\psi(y)+\int K(x, y)(\nu+\bar{y})(d x)\right]
$$

Now $\exp [-\psi / 2]$ is the characteristic functional of a centered Gaussian cylinder measure $\gamma$ on $E$; also, by Lemma 4.2, there exists a cylinder measure $\varrho$ on $E$ such that $\hat{\varrho}=\exp \left[\int K(x, \cdot) \nu(d x)\right]$. Equation (7) implies that the cylincler measures $\mu * \bar{\mu}, \gamma * \gamma$ and $\varrho * \bar{\varrho}$ satisfy

$$
\mu * \bar{\mu}=(\gamma * \gamma) *(\varrho * \bar{\varrho}) \text {. }
$$

Since $\mu * \bar{\mu}$ is a measure on $E$, it follows from Lemma 4.1 that $\gamma * \gamma$ and $\varrho * \bar{Q}$ are measures on $E$. But $\gamma * \gamma=\gamma\left(2^{-1 / 2}(\cdot)\right)$; hence $\gamma$ is a measure on $E$

Let us return to (2). Passing to the limit along the subsequence $\left\{n_{k}\right\}$ Tre get, by a well-known elementary argument: there exists a linear form $f$ $E^{\prime} \rightarrow R^{1}$ such that $f_{n_{k}}(y) \rightarrow f(y)$ for all $y \in E^{\prime}$ and

$$
\hat{\mu}(y)=\exp \left[i f(y)-(1 / 2) g(y)+\int B(x, y) v(d x)\right]
$$

$$
\hat{\mu}(y)=\exp \left[i f(y)-(1 / 2) \psi(y)+\int K(x, y) v(d x)\right] .
$$

Now $\hat{\mu}, \exp [-\psi / 2]$ and $\hat{\varrho}$ are sequentially $w^{*}$-continuous (the first two, because they are characteristic functionals; the third, by Lemma 4.2). It follows that $f$ is a sequentially $w^{*}$-continuous linear form on $W^{\prime}$; therefore there exists $x_{0} \in E$ such that $f(y)=\left\langle x_{0}, y\right\rangle, y \in E^{\prime}([17]$, p. 150).

Equation (8) implies that the cylinder measures $\mu, \delta_{x_{0}}, \gamma$ and $\varrho$ satisfy

$$
\mu=\left(\delta_{x_{0}} * \gamma\right) * \varrho .
$$


Since $\mu$ and $\left(\delta_{s_{0}} * \gamma\right)$ are measures on $E$, so is $\varrho$ by Lemma 4.1. This proves the existence statement of the theorem. We omit the proof of wniqueness; it is carried out as in ([16], p. 110). Let us remark that the proof of uniqueness is valid in any separable Banach space; the cotype 2 condition is not used.

(2) Let $\sigma$ be the Poisson distribution with parameter 1 , and let $\left\{\varrho_{j}: j \in N\right\}$ be a sequence of independent random variables with $\mathscr{L}\left(\varrho_{j}\right)$ $=\sigma * \bar{\sigma} \quad(j \in N)$. We first prove: $\sum_{j} e_{j} x_{j}$ converges in $L^{2}(E)$ implies $\sum_{j}\left\|x_{j}\right\|^{2}$ $<\infty$. In fact, suppose $X=L^{2}(E)-\lim _{n} \sum_{j=1}^{n} \varrho_{j} x_{j}, \quad \mu=\mathscr{L}(X)$, and let $\lambda=\sum_{j}\left(\delta_{x_{j}}+\delta_{\left(-x_{j}\right)}\right)$. Then

$$
\begin{aligned}
\hat{\mu}(y) & =\prod_{j=1}^{\infty} \mathscr{L}\left(\varrho_{j} x_{j}\right)^{\wedge}(y)=\exp \left[\int[\operatorname{cxp}(i\langle x, y\rangle)-1] \lambda(d x)\right] \\
& =\exp \left[\int K(x, y) \eta(d x)\right]
\end{aligned}
$$

where

$$
\eta(d x)=\frac{\|x\|^{2}}{1+\|x\|^{2}} \lambda(d x) .
$$

Since $\mu$ is infinitely divisible, by hypothesis there exist a point $x_{0} \in E$, a centered Gaussian measure $\gamma$ on $E$ and a finite non-negative measure $\nu$ with $\nu(\{0\})=0$ on $E$, such that

$$
\hat{\mu}(y)=\exp \left[i\left\langle x_{0}, y\right\rangle-(1 / 2) \psi(y)+\int K(x, y) v(d x)\right] .
$$

The uniqueness of the representation implies $x_{0}=0, \psi=0, v=\eta$. Therefore

$$
2 \sum_{j} \frac{\left\|x_{j}\right\|^{2}}{1+\left\|x_{j}\right\|^{2}}=\int \frac{\|x\|^{2}}{1+\|x\|^{2}} \lambda(d x)=\eta(E)=v(E)<\infty
$$

and consequently $\sum_{j}\left\|x_{j}\right\|^{2}<\infty$.

By an argument based on the closed graph theorem and analogous to that given in [10], p. 588, it follows that there exists a constant $O>0$ such that for every fimite set $\left\{x_{1}, \ldots, x_{n}\right\} \subset E$,

$$
\sum_{j=1}^{n}\left\|x_{j}\right\|^{2} \leqslant C E\left\|\sum_{j=1}^{n} \varrho_{j} x_{j}\right\|^{2}
$$

In order to complete the proof that is of cotype 2 , it is enough to prove that $c_{0}$ is not finitely representable in $E$ ([15], p. 49). For the proof that $(\mathrm{a}) \Rightarrow(\mathrm{b})$ in Corollary 1.2, p. 67, of [15] then shows that there exists a con- stant $M>0$ such that for every finite set $\left\{x_{1}, \ldots, x_{n}\right\} \subset E$,

$$
E\left\|\sum_{j=1}^{n} \varrho_{j} x_{j}\right\|^{2} \leqslant M E\left\|\sum_{j=1}^{n}{ }^{n} \varepsilon_{j} x_{j}\right\|^{2} .
$$

Inequalities (9) and (10) show that $E$ is of cotype 2.

Suppose now that $c_{0}$ is finitely representable in $E$. By ([15], p. 50, Remarque 0.2$)$, for etery $n \in N$ there exists an $n$-uple $\left(x_{1}^{(n)}, \ldots, x_{n}^{(n)}\right)$ of vectors in $D$ with $\left\|x_{3}^{(n)}\right\| \geqslant 1 / 2$ such that for every sequence $\left(a_{j}\right)_{j \in N}$ in $R^{1}$.

$$
\| \sum_{j=1}^{n} \alpha_{j} x_{j}^{(n)}|| \leqslant \sup _{1 \leqslant j \leqslant n}\left|\alpha_{j}\right|
$$

Putting $a_{j}=a_{j} \varrho_{j}$ with $a_{j} \in R^{l}$, taking expectations and combining (9) and (11), we obtain

$$
\text { (1/4) } \sum_{j=1}^{n}\left|a_{j}\right|^{2} \leqslant \sum_{j=1}^{n}\left\|a_{j} x_{j}^{(n)}\right\|^{2} \leqslant O E\left\|\sum_{j=1}^{n} a_{j} \varrho_{j} x_{j}^{(n)}\right\|^{2} \leqslant O E\left(\sup _{1 \leqslant j \leqslant n}\left|a_{j} \varrho_{j}\right|\right)^{2} .
$$

Since $E\left(\sup _{1 \leqslant j \leqslant n}\left|a_{j} \varrho_{j}\right|\right)^{2} \leqslant\left(E\left|\varrho_{1}\right|^{p} \sum_{j=1}^{n}\left|a_{j}\right|^{p}\right)^{2 / 2}$ ( $p$ any fixed real number $\left.>2\right)$, a contradiction is obtained by appropriate choice of $\left(a_{j}\right)_{j \in N}$.

Note. A complete characterization of the Gaussian covariance $\psi$ by continuity properties has been obtained by $\mathrm{E}$. Gine and the firstnamed author. This will appear elsewhere.

Acknowledgement. We thank A. de Araujo and E. Giné for some stimulating conversations, and G. Pisier for pointing out an error in a previous version and directing our attention to [15].

\section{References}

[1] A. de Araujo, On infinitely divisible laws in $O[0,1]$, Proc. Amer. Math. Soc. 61 (1975), pp. 179-185.

[2] - and E. Giné, Type, cotype and Lévy measures in Banaeh spaces, Ann. of Probab. 6, 4 (1978), pp. 637-643.

[3] A. Badrikian, Séminaire sur les fonctions aléatoires linéaires et les mesures eylindriques, Lect. Notes in Matl. 139, Springer-Verlag, Berlin and New York 1970.

[4] K. L. Chung, A course in probability theory (2-nd. edition), Academio Press, New York 1974.

[5] Ph. Courrège, Génerateur infinitésimal d'un semigroupe de convolution sur $R^{n}$ et formule de Lévy -Khintohine, Bull. Sei. Math., 2-ème. série, 88 (1964), pp. 3-30.

[6] E. Dettweiler, Grenzwertsätze für Wahrseheinlichkeitsmasse auf Badrikianschen Räumen, Z. Wahrscheinlichkeitstheorie verw. Gebiete 34 (1976), pp. 285-311.

[7] W. Feller, An introduction to probability theory and its applications, Vol. II (2-nd. edition), Wiley, Now York 1970. 
[8] B. F. Gnedenko and A. N. Kolmogorov, Limit distribution for sums of independent random variables, Addison Wesley, Reading, Mass. 1968.

[9] J. Hoffmann-Jorgensen, Sums of independent Banach space valued random variables, Studia Math. 52 (1974), pp. 159-186.

[10] - and G. Pisier, The law of large numbers and the central limit theorem in Banach spaces, Ann. of Probab. 4, 4 (1976), pp. 587-599.

[11] K. Ito, and M. Nisio, On the convergence of sums of independent Banach space valued random variables, Osaka J. Math. 5 (1968), pp. 35-48.

[12] N. Jain, and M. Marcos, Integrability of infinite sums of independent vector. valued random variables, Trans. Amer. Math. Soc. 212 (1975), pp. 1-36.

[13] J. P. Kahane, Some random series of functions, Heath, Lexington, Mass. 1968.

[14] L. Le Cam, Remarques sur le théoreme limite centrale dans les espaces localement convexes. Les Probabilités sut les structures algébriques, C.N.R.S., Paris 1970, pp. $233-249$.

[15] B. Mauxey and G. Pisier, Séries de variables aléatoires vectorielles indépendents et proprietés géométriques des espaces de Banach, Studia Math. 58 (1976), pp. $45-90$.

[16] I. R. Parthasarathy, Probability measures on metrie spaces, Academic Press, New York 1967.

[17] H. H. Schaefer, Topological vector spaces, Mac Millan, New York 1966.

[18] A. Tortrat, Structure des lois indéfiniment divisibles dans un espace vectoriel topologique, Lecture Notes in Math. 31 (1967), pp. 299-328.

[18a] - Sur la structure des lois indéfiniment divisibles dans les espaces vectoriels, Z. Wahrscheinlichkeitstheorie verw. Gebiete Il (1969), pp. 311-326.

[19] S. R. S. Varadhan, Limit theorems for sums of independent random variables with ralues in a Hilbert space, Sankhya 24 (1962), pp. 213-238.

INSTITUTO VENEZOLANO DE INVESTIGACIONES CIENTIFICAS OARACAS, VENEZUELA.

and

UNTTERSTDAD NACTONAI DE LA PLATA,

LA PLATA, ARGENTINA

\section{Multiply self-decomposable probability measures} on Banach spaces

by

\section{NGDYEN VAN THU (Wroclaw)}

Abstract. In the present paper we define multiply self-decomposable probability measures on a Banach space and give a general form of their characteristic functionals.

1. Introduction. This paper is concerned with probability measures defimed on Borel subsets of a real separable Banach space $\bar{X}$. For a probability measure $\mu$ on $X$, the characteristic functional $\hat{\mu}$ is defined on the dual space $X^{*}$ by the formula

$$
\hat{\mu}(y)=\int_{x} e^{i\langle u, x\rangle} \mu(d x) \quad\left(y \in X^{*}\right),
$$

where $\langle\cdot, \cdot\rangle$ denotes the dual pairing between $X$ and $X^{*}$.

Recall that a probability measure $\mu$ on $X$ is self-decomposable if for every number $c$ in $(0,1)$ there exists a probability measure $\mu_{c}$ on $X$ such that

$$
\hat{\mu}(y)=\hat{\mu}(c y) \hat{\mu}_{c}(y) \quad\left(y \in X^{*}\right)
$$

The problem of describing the class of characteristic functionals of self-decomposable probability measures has been completely solved by Urbanik [8]. In the same paper the author has obtained a general form of characteristic functionals even for a larger class of probability measures, namely, for Levy's measures on $X$.

We now introduce a concept of multiply self-decomposable probability measures on Banach spaces. Let $L_{1}(X)$ denote the class of all self-decomposable probability measures on $X$. For every integer $n>1$, let $L_{n}(X)$ denote the class of all measures $\mu$ in $L_{1}(X)$ such that for every number 0 in $(0,1)$ the component $\mu_{c}$ in $(1.1)$ belongs to $L_{n-1}(X)$. Every measure in $L_{n}(X)$ will be called n-times self-decomposable. Further, every measure in $L_{\infty}(X):=\bigcap_{n=1}^{\infty} L_{n}(X)$ will be called completely self-decomposable. Since every stable measure on $X$ is completely self-decomposable (Proposition 1.9, [3]), the set $L_{\infty}(X)$ is non-empty. 